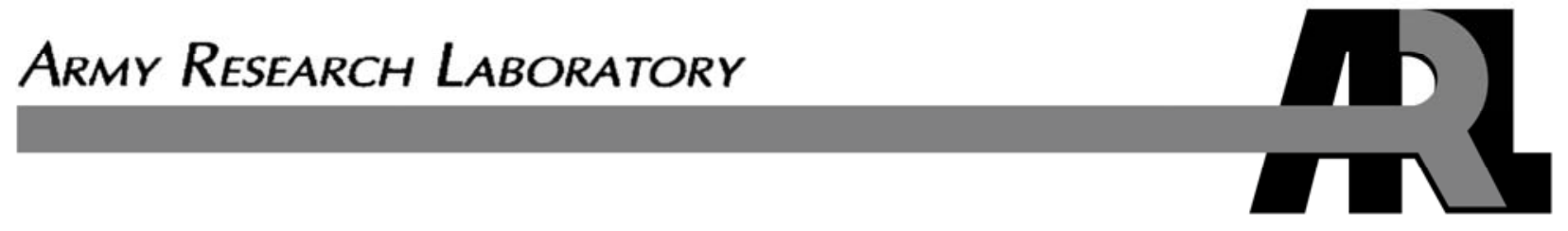

\title{
Soldier-Robot Teaming: Effects of Multimodal Collaboration on Team Communication for Robot Reconnaissance
}

by Shaun Hutchins, Keryl Cosenzo, Michael Barnes, Theodric Feng, and Krishna Pillalamarri

ARL-TR-5385

October 2010 


\section{NOTICES}

\section{Disclaimers}

The findings in this report are not to be construed as an official Department of the Army position unless so designated by other authorized documents.

Citation of manufacturer's or trade names does not constitute an official endorsement or approval of the use thereof.

Destroy this report when it is no longer needed. Do not return it to the originator. 


\title{
Army Research Laboratory
}

Aberdeen Proving Ground, MD 21005-5425

\section{Soldier-Robot Teaming: Effects of Multimodal Collaboration on Team Communication for Robot Reconnaissance}

\author{
Shaun Hutchins \\ Alion Science and Technology Inc.
}

Keryl Cosenzo, Michael Barnes, Theodric Feng, and Krishna Pillalamarri Human Research and Engineering Directorate, ARL 


\section{REPORT DOCUMENTATION PAGE}

Form Approved OMB No. 0704-0188

Public reporting burden for this collection of information is estimated to average 1 hour per response, including the time for reviewing instructions, searching existing data sources, gathering and maintaining the data needed, and completing and reviewing the collection information. Send comments regarding this burden estimate or any other aspect of this collection of information, including suggestions for reducing the burden, to Department of Defense, Washington Headquarters Services, Directorate for Information Operations and Reports (0704-0188), 1215 Jefferson Davis Highway, Suite 1204, Arlington, VA 22202-4302. Respondents should be aware that notwithstanding any other provision of law, no person shall be subject to any penalty for failing to comply with a collection of information if it does not display a currently valid OMB control number.

PLEASE DO NOT RETURN YOUR FORM TO THE ABOVE ADDRESS.

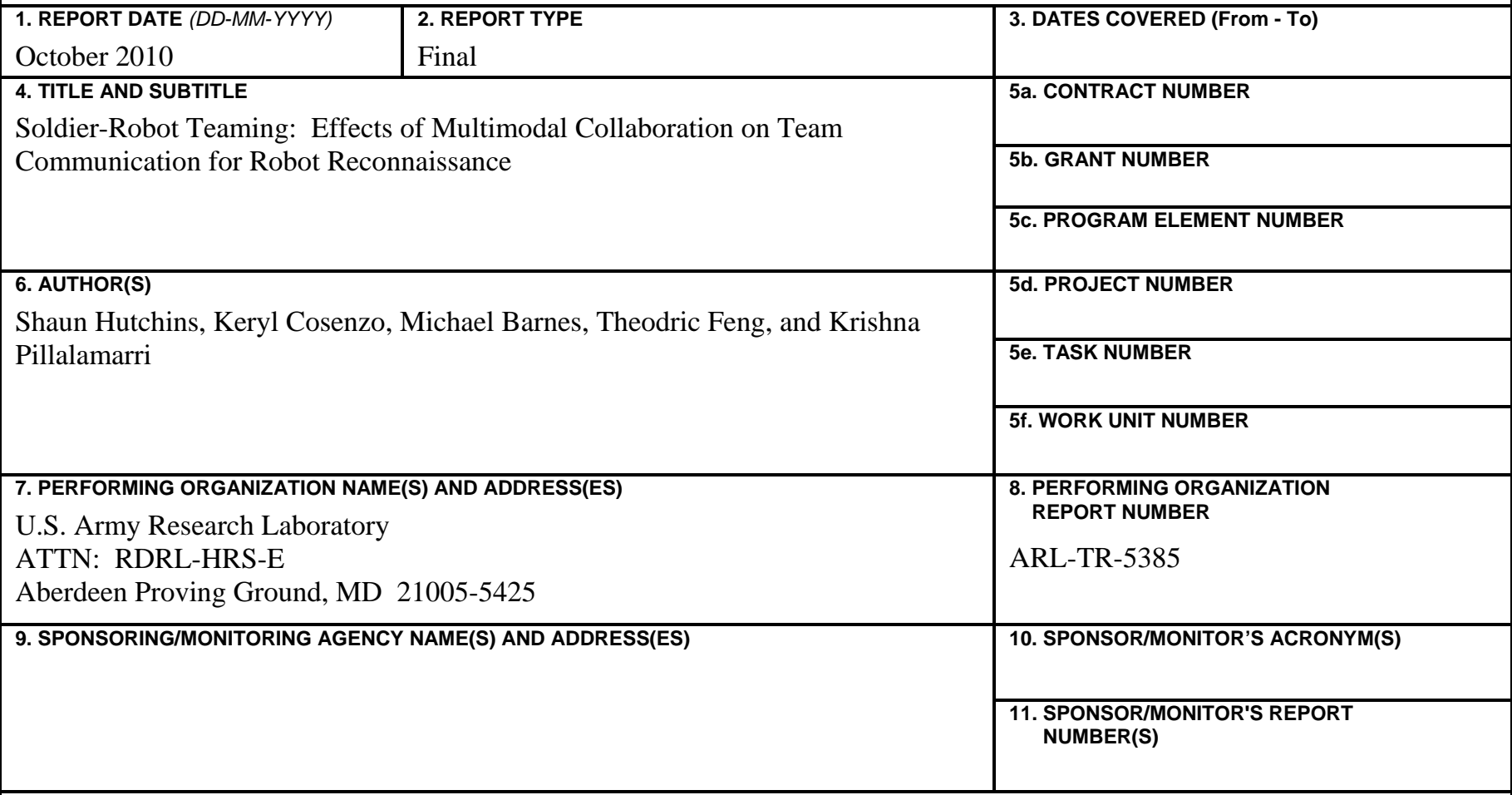

\section{DISTRIBUTIONIAVAILABILITY STATEMENT}

Approved for public release; distribution unlimited.

\section{SUPPLEMENTARY NOTES}

\section{ABSTRACT}

The impacts on performance of three different forms of communication (radio, chat, and tactile belt) were explored in the context of a small unmanned ground vehicle (SUGV) target identification task. The target identification task required a commander with knowledge of target locations and access to a digital map displaying the current SUGV position and orientation to direct a Soldier remotely operating the SUGV to the targets using a finite set of 11 commands. The study revealed no evidence of a loss of Soldier performance using the tactile belt communications channel. The finding suggests that the tactile use of haptic signals may be feasible, a potentially important finding for situations requiring covert communications.

\section{SUBJECT TERMS}

multimodal displays, robotics, field experiment

\section{SECURITY CLASSIFICATION OF:}

a. REPORT

Unclassified

b. ABSTRACT

Unclassified

\section{c. THIS PAGE}

Unclassified

\begin{tabular}{l|c} 
17. LIMITATION & 18. NUMBER \\
OF & OF \\
ABSTRACT & PAGES \\
UU & 30
\end{tabular}

19a. NAME OF RESPONSIBLE PERSON

Shaun Hutchins

19b. TELEPHONE NUMBER (Include area code) (410) 278-5885 


\section{Contents}

$\begin{array}{ll}\text { List of Figures } & \text { v }\end{array}$

List of Tables $\quad$ v

$\begin{array}{lr}\text { 1. Introduction } & 1\end{array}$

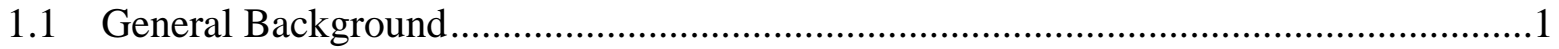

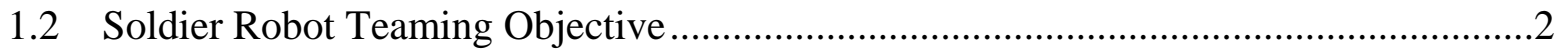

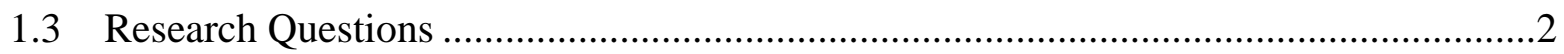

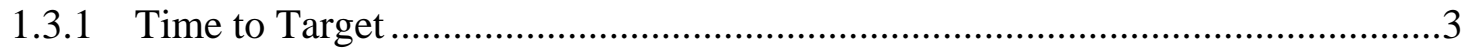

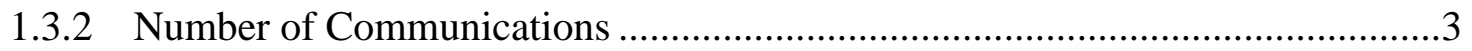

2. Method 3

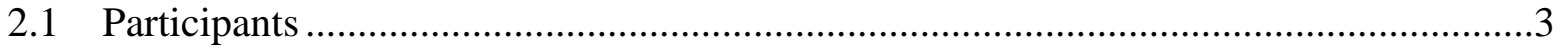

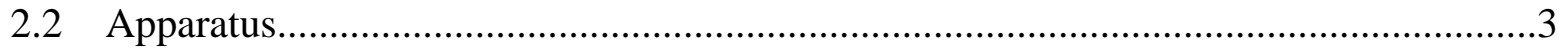

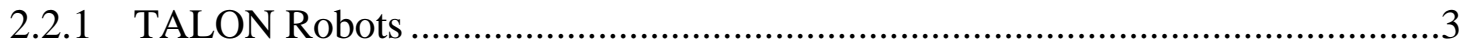

2.2.2 AMREL Dismounted Controller ..................................................................4

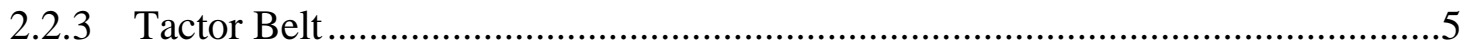

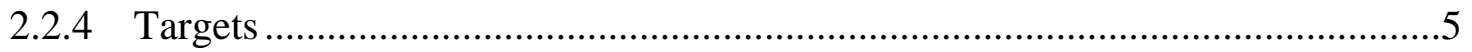

2.2 .5 Questionnaires ...................................................................................

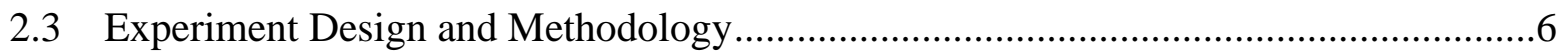

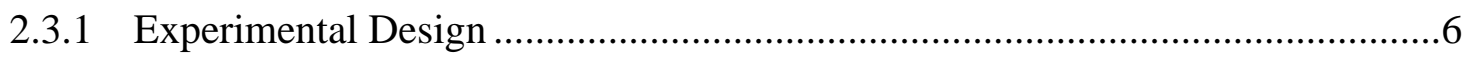

2.3.2 Experimental Dismount Team.............................................................6

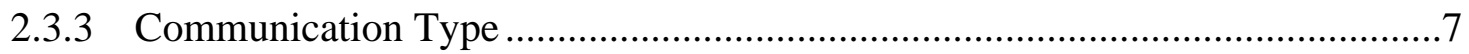

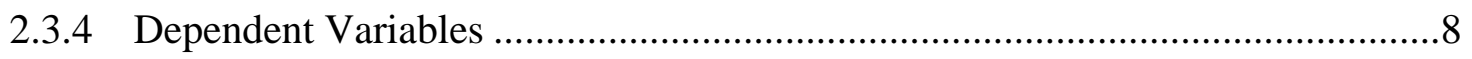

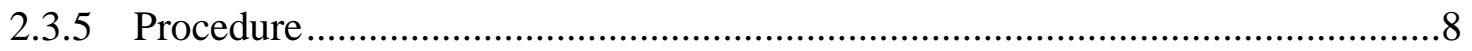

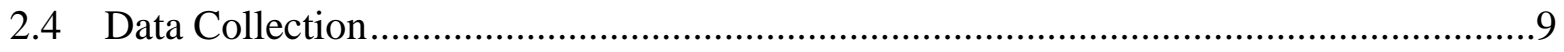

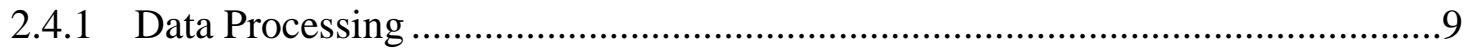

3. Analyses and Results $\quad 10$

3.1 Time to Identify Targets................................................................................. 10

3.1.1 Data Screening and Selection ................................................................. 10

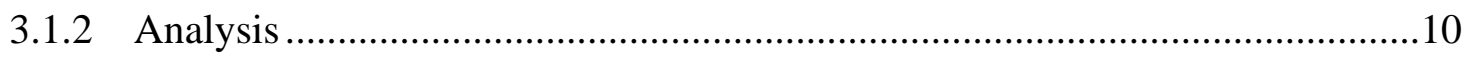

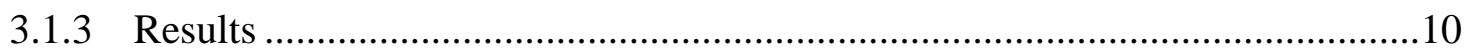


3.2 Number of Navigational Communications.........................................................11

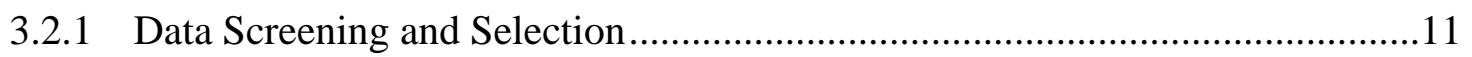

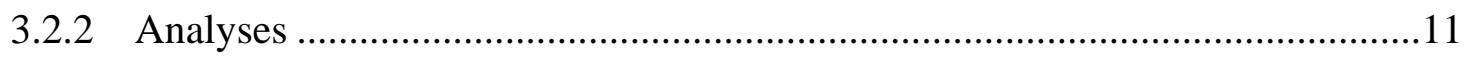

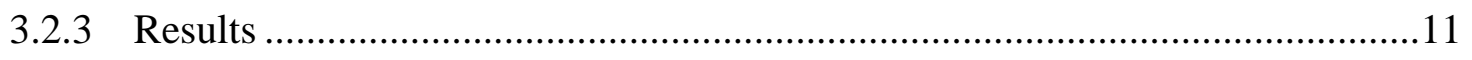

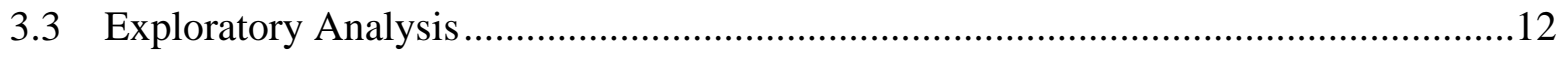

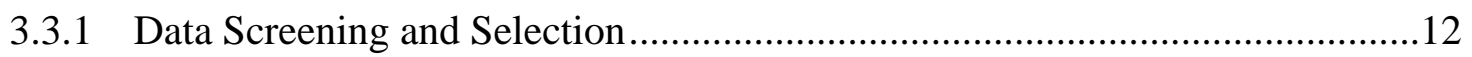

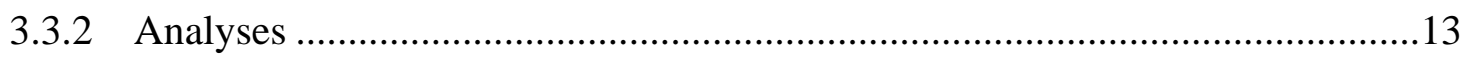

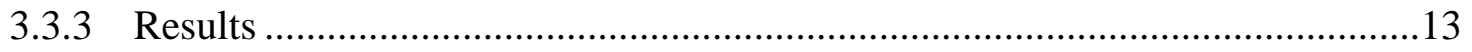

3.4 NASA TLX and SART Analyses...................................................................13

3.4.1 Data Screening and Selection ................................................................13

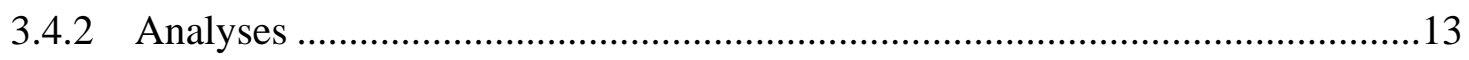

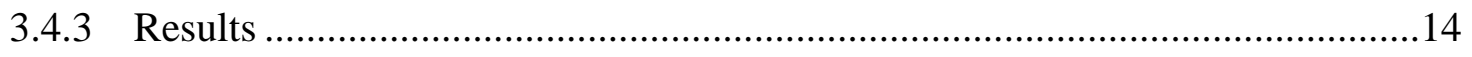

$\begin{array}{lr}\text { 4. Conclusion } & 14\end{array}$

5. References $\quad 16$

$\begin{array}{lr}\text { List of Symbols, Abbreviations, and Acronyms } & 18\end{array}$

$\begin{array}{ll}\text { Distribution List } & 19\end{array}$ 


\section{List of Figures}

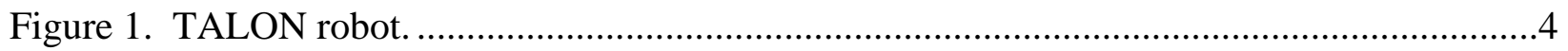

Figure 2. AMREL dismounted controller......................................................................4

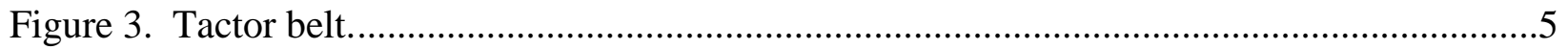

\section{List of Tables}

Table 1. Randomly assigned exposure orders of communication conditions and course

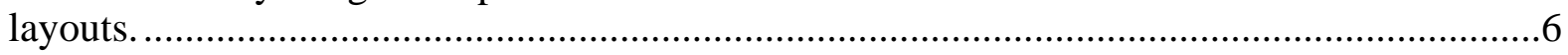

Table 2. Post-hoc tests on time to identify (seconds) by target type. ......................................11

Table 3. Communication count pos- hoc tests on target type.................................................12

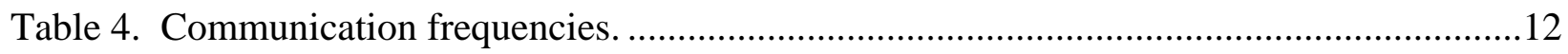

Table 5. Time to identify targets: median split.....................................................................13

Table 6. Communication frequency median split. ................................................................13 
INTENTIONALLY LEFT BLANK. 


\section{Introduction}

In 2004, the U.S. Army Tank-Automotive Research Development and Engineering Center (TARDEC), in partnership with the U.S. Army Research Laboratory (ARL) Human Research and Engineering Directorate (HRED), pursued a 5-year program U.S. Army Technology Objective (ATO). The purpose of the ATO was to develop the tools, techniques, and autonomy to maximize mounted and dismounted control of ground and air unmanned systems and optimize Soldier-robot and robot-robot ground and air teams. Development included a scalable user interface for robotic control. The interface maximizes multi-function Soldier performance for primary tasks while minimizing unique training requirements, achieved by optimizing and standardizing the required interactions and managing the workload associated with the control of unmanned ground and air systems. This report highlights the Robotics Collaboration ATO Capstone Experiment on small robot control.

\subsection{General Background}

As the emerging technologies of the Army's Future Combat System (FCS) are introduced to the battlefield, Soldiers will increasingly face new challenges in workload management. A shifting force structure will bring increasing responsibilities for the next generation Soldier, who will be tasked with effectively using and protecting robotic assets in addition to performing other primary missions. TARDEC and HRED are addressing issues in human-robot interaction (HRI) under the Robotics Collaboration (RC) ATO program. The key program goal is to understand HRI issues in order to develop technologies and mitigations that enhance HRI performance in future combat environments.

Robots have been and continue to be effective in combat tasks that are "dull, dirty, and dangerous” (Carafano and Gudgel, 2007). Remotely controlled robotic unmanned air vehicles (UAVs) and unmanned ground vehicles (UGVs) play a vital role in current operations, including disarming roadside bombs and performing ground- and air-based patrol missions. However, these systems involve the cognitive resources of the operators, who are continuously engaged in teleoperating the systems and manipulating the sensors or payloads during the mission. Future military operations require the Soldier to be as unencumbered by equipment and weapon constraints as possible. At the same time, the number of possible systems that are available at even the squad level will increase dramatically from the current force. In particular, the use of robotic systems will enhance the operator's safety, flexibility, and lethality during urban missions-but at a price. Performance is enhanced by the ability to remotely "see" and "shoot" in buildings, disarm explosive devices, and view around corners. The price is the additional workload and loss of situation awareness (SA) when the Soldier is most vulnerable. Robotic teleoperation focuses the operator on the robotic task, to the detriment of personal security (Chen and Joyner, 2009). 
The twin goals of the Soldier-robot teaming research were to understand HRIs in a military environment and assess technologies that mitigate undesirable effects. One area of interest was the use of innovative solutions such as multimodal interfaces. In general, ARL researchers found that using tactile and auditory cueing both separately and as redundant cues improved Soldier multitasking performance (Chen, Haas, and Barnes, 2007; Chen and Terrence, 2008; Haas, 2007). Tactile cueing was found to be more flexible because it did not require the Soldier to disambiguate between front and back signals for navigation tasks. Perhaps more importantly, tactile cueing uses communications channels that are currently underused in military environments (Barnes, in press). For example, our research verified that tactile information could be used for covert communications using a limited lexicon that was easily learned by Soldier participants (Gilson, Redden, and Elliott, 2007). Ease of learning tactile cues was a reoccurring trend in many of the published studies, further suggesting the advantages of tactile communications for Soldier robot teaming operations (Jones and Sarter, 2008).

For example, Pettit, Redden, and Carstens (2006) translated Army hand and arm signals to a covert tactile communication system, resulting both in improved navigation aids and as a silent signaling environment for army snipers (Gilson et al., 2007). However, the potential uses of covert, low-bandwidth tactile modes go beyond communication networks among Soldiers. As robots develop more environmental awareness, tactile communications might be possible as a two-way conduit between robots and Soldier teams (Barnes, in press). The current study was designed to explore this possibility in a realistic field environment at Ft. Bliss in El Paso, TX, with experienced Soldiers. Soldiers conducted search missions in desert environment, not too unlike the Iraqi desert. The Soldier operators were given minimal training using a TALON robot with three communication modes: chat (visual text), radio, and tactile. The Soldier operator was directed by a remotely located commander to target locations hidden in the desert terrain. The operator in turn directed the TALON robot to various locations using a specially designed interface based on the commander's inputs in one of the three communications modes. This emulated Soldier teams or Soldier robot teams working in close concert and sharing SA.

\subsection{Soldier Robot Teaming Objective}

The objective of the current research was to explore potential relationships between modes of communication, i.e., auditory, visual, or tactile, and task performance for shared control of dismounted operations with a small unmanned ground vehicle (SUGV). Specifically, the relationships were explored in a SUGV teleoperation task in which critical navigation and map information was the focus of the dismount team's shared control.

\subsection{Research Questions}

This research sought to address the impact of the type of communication, i.e., auditory, visual, or tactile, on a dismounted team's performance on a target identification task. To help answer the overall question, several specific questions were addressed in the experiment. 


\subsubsection{Time to Target}

Are there differences between the three levels of communications type (radio, chat, and tactile) with regard to the average time to identify a target? Are there differences between the three levels of target type (large, medium, and small) with regard to the average time to identify a target? Is there an interaction between communications type and target type with regard to the average time to identify a target?

\subsubsection{Number of Communications}

Are there differences between the three levels of communications type (radio, chat, and tactile) with regard to the average number of communications required to identify a target? Are there differences between the three levels of target type (large, medium, and small) with regard to the average number of communications required to identify a target? Is there an interaction between communications type and target type with regard to the average number of communications required to identify a target?

We hypothesized that the tactile communications would result in improved latency and accuracy performance compared to traditional communication modes (i.e., radio).

\section{Method}

\subsection{Participants}

Volunteer participants consisted of 13 active-duty Soldiers from the 2nd Combined Arms Battalion 5th Brigade (Army Experiment Task Force) located at Ft. Bliss, TX. All of the Soldiers were male ranging in age from 20-31 with an average of 24.08 years of age.

\subsection{Apparatus}

\subsubsection{TALON Robots}

The SUGV for this experiment was the TALON robot. The TALON is a man-portable (45 kg) rugged robot that can negotiate through all types of terrain and can withstand all types of weather (figure 1). 


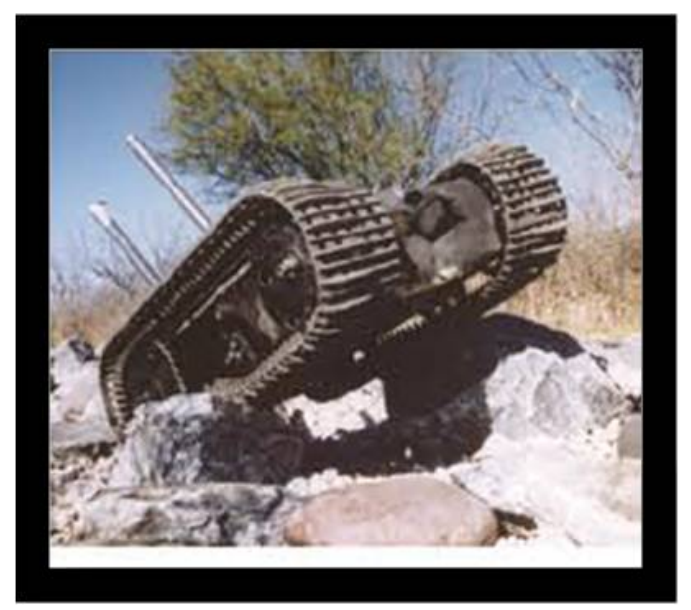

Figure 1. TALON robot.

\subsubsection{AMREL Dismounted Controller}

The controller of the SUGV allows the dismounted Soldier to operate the SUGV and share information with his team member about mission-critical events. It is a tablet-based controller (figure 2) with a resistive touch screen and a stylus that is ruggedized for the extreme weather conditions and suited with military connection capability. The controller has the following dimensions:

- 9.8 in wide $\times 7.4$ in diameter $\times 1.65$ in high

- $4.2 \mathrm{lb}$ with primary battery

- $4.7 \mathrm{lb}$ with second battery

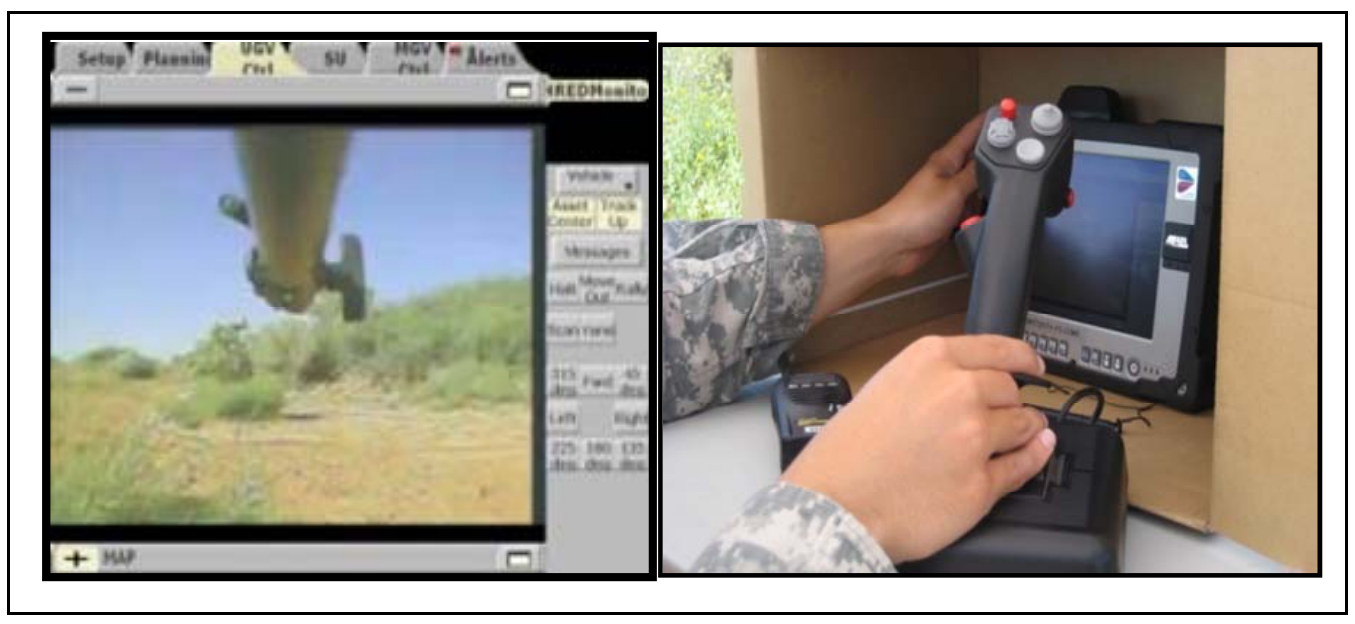

Figure 2. AMREL dismounted controller. 


\subsubsection{Tactor Belt}

The tactor belt was worn around the waist and contained eight individual tactors (the C-2 model, Engineering Acoustics, Inc.; figure 3). The group of tactors functioned together and vibrated in a unique pattern to distinguish discrete commands.

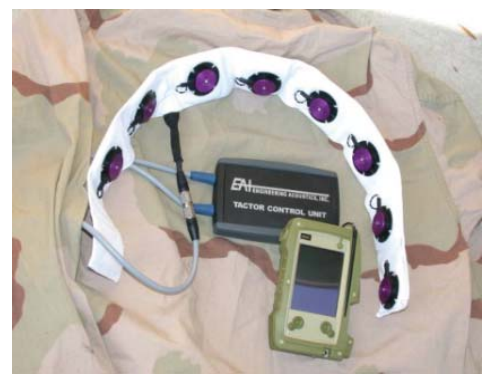

Figure 3. Tactor belt.

\subsubsection{Targets}

Large, medium, and small targets were used in each experimental mission. A mission was the experimental course. The large target was a construction barrel $\sim 4 \mathrm{ft}$ high. The medium target was an orange cone $\sim 18$ in high. The small target was a white pipe $\sim 12$ in long.

\subsubsection{Questionnaires}

Subjective workload and SA ratings were measured with the National Aeronautics and Space Administration-Task Load Index (NASA-TLX) (Hart and Staveland, 1987) and the Cognitive Compatibility Situation Awareness Technique Questionnaire (CC-SART) (Taylor, 1990), respectively. The NASA-TLX is a multi-dimensional rating procedure that derives an overall workload score based on a weighted average of ratings on six subscales (Mental Demand, Physical Demand, Temporal Demand, Own Performance, Effort, and Frustration). The CCSART is a subjective rating of SA, in which participants rate their experience with the task on three dimensions (Activation of Knowledge, Ease of Reasoning, and Level of Processing). Level of Processing is the degree to which the situation involves, at the low level, natural automatic, intuitive, and associated processing or, at the high level, analytic, considered, conceptual, and abstract processing. Ease of Reasoning is the degree to which the situation, at a low level, is confusing and contradictory or, at a high level, is straightforward and understandable. Activation of Knowledge is the degree to which the situation, at the low level, is strange and unusual or, at the high level, is recognizable and familiar. Workload and SA were assessed at the end of each mission. 


\subsection{Experiment Design and Methodology}

\subsubsection{Experimental Design}

The experiment was a $3 \times 3$ within-subject design. Communication type and target type were two independent variables of experimental interest. Communication type had three categories: radio (audio), chat (visual), and tactile. Target type had three levels (large, medium, and small). Participants completed two missions in each of the three communication conditions; therefore, each participant completed six missions. A mission consisted of the participant driving the robot through a course with embedded targets (one large target, one medium target, and two small targets) that he had to identify.

There were three courses used in the experiment. Due to logistical constraints, the three courses were set up prior to the experiment and target locations were fixed for the duration of the experiment. The size of the course areas, the terrain features, and the distances between targets within each course were approximately equivalent between the courses. On each course there were four targets randomly dispersed in the course area. So in one course the first target could be a small target and in another course the first target could be a large target. Participants ran the robot through each course in succession, course 1 , course 2 , and then course 3 . This sequence was repeated for a total of six missions. Table 1 shows the randomly assigned order of exposure to the various communication conditions and course layouts.

Table 1. Randomly assigned exposure orders of communication conditions and course layouts.

\begin{tabular}{|c|c|c|c|c|c|c|}
\hline & Mission 1 & Mission 2 & Mission 3 & Mission 4 & Mission 5 & Mission 6 \\
\hline Participants & Course 1 & Course 2 & Course 3 & Course 1 & Course 2 & Course 3 \\
\hline $1,12,13$ & Radio & Radio & Chat & Chat & Tactor & Tactor \\
\hline 2,11 & Tactor & Tactor & Chat & Chat & Radio & Radio \\
\hline 3,10 & Radio & Radio & Tactor & Tactor & Chat & Chat \\
\hline 4,9 & Tactor & Tactor & Radio & Radio & Chat & Chat \\
\hline 5,8 & Chat & Chat & Radio & Radio & Tactor & Tactor \\
\hline 6,7 & Chat & Chat & Tactor & Tactor & Radio & Radio \\
\hline
\end{tabular}

The three types of communication conditions produced a total of six possible exposure orders. Repetitions of the six potential exposure orders to the three communications conditions was balanced to the best extent possible across 13 participants such that two of the exposure orders were assigned two repetitions and one was allowed 3 repetitions. The assignment of participant to exposure condition was random without replacement. Radio, then chat, and then tactors was the exposure order with three randomly assigned participants.

\subsubsection{Experimental Dismount Team}

The experimental team consisted of a commander, a robotic controller, and a robotic follower. The role of the commander was filled by an experimental team member. The commander was in charge of relaying navigational commands to the robotic controller, directing him towards the 
targets. The role of the robotic controller was filled by a Soldier participant. The robotic controller was in control of the TALON mobility and responsible for identified targets. The role of the robotic follower was filled by an experimental team member. The robotic follower was responsible for the safety of the TALON while on the course.

\subsubsection{Communication Type}

Three communication modalities were used in the study: Chat interface, tactor belt, and radio. The chat, or visual, modality provided the participant (i.e., robotic controller) and the commander with a suite of communications on the AMREL tablet. A discrete set of communications were represented with interface buttons, one button per communication. Selecting one of the buttons in the suite sent the representative chat message to the team member and was displayed as a text message on the AMREL tablet. The tactor belt contained eight individual tactors. The tactors functioned together and vibrated in a unique pattern for each of the discrete communications. As in the chat condition, selecting one the buttons in the suite of discrete communications on the AMREL tablet sent the representative message to the team member; however, in this condition, the team member's belt would vibrate according to the type of message sent. The radio condition, or auditory modality, used hand-held two-way radios. All communications were shared using the radios, but the types of communications were restricted to the same set of discrete communications that were available in the chat and tactor conditions. The commander and the robotic operator sent and received communications via the AMREL displays by using either tactile belt (wore by commander and operator) or chat messaging.

Communication between the commander and the participant in the dismount team was two way within the communication modality of the condition. The commander in the dismount team had a digital map on his AMREL display showing the current SUGV position (from GPS) and orientation as well as target locations. The commander directed the participant with navigational commands. The participant teleoperated the TALON to the critical areas based on the commander's direction; however, the commander did not "micromanage" the operator (i.e., specify which way to go around bushes). Using the condition-appropriate communication modality, the commander indicated when the robot was in the target vicinity and the participant would scan to find the target. Commands were limited to a discrete set of 11 communications:

- $\quad$ Scan forward

- Scan left

- Scan right

- Transit $180^{\circ}$

- Transit forward

- $\quad$ Transit left 
- Transit right

- Found

- Halt

- Rally

- Move out

\subsubsection{Dependent Variables}

Two primary dependent measures were collected: time to identify targets and the total number of navigational communications.

- Time to identify targets was defined as the number of seconds from when the communication to "move out" was given at the beginning of a course segment until the "found" communication was given upon identifying a target.

- Number of navigational communications was defined as the frequency of navigation communications issued from when the communication to "move out" was given at the beginning of a course segment until the "found" communication was given upon identifying a target.

\subsubsection{Procedure}

The participant was verbally briefed on his role in the teleoperation tasks in the experiment. The brief included a description of the modes of communication and target types central to the experimental tasks, as well as a task demonstration. The Soldier then practiced the experimental tasks with each communication modality on a separate training course with targets. Practice was structured such that a communication modality was first demonstrated to the Soldier, and then the Soldier completed a training run within that communication modality, repeating this practice structure across all three communication conditions. Radio was always introduced first, then chat, and lastly tactor. The exception to this practice procedure was for the tactor communication modality. The Soldier experienced every communication condition multiple times, and then recognition of communications was tested until 100\% accuracy for identifying communications was achieved. This recognition test was repeated later, just prior to beginning the tactor experiment trials for further practice and competence.

The Soldier then began the sequence of experimental missions. Each participant completed one mission in a single modality, and then repeated the mission in the same modality a second time. The two repetitions of each of the three modalities yielded a total of six missions per participant. Immediately following the first pair of sequential missions within a single communications modality, the Soldier was administered the NASA-TLX and SART questionnaires. The Soldier performed the second pair of missions followed with associated questionnaires; and lastly the third pair of missions with associated questionnaires. Within a mission, the procedure was to 
first get verbal confirmation from the Soldier of readiness to begin. The commander then issued a "move out" command to begin the mission, followed by a series of additional navigational commands directing the Soldier to each target within the mission. The participant reported "Target found" upon identifying each target and the commander would respond with a "Confirm." After receiving the confirmation, the Soldier progressed to the next target in the mission. Upon identifying the fourth target in the mission, the commander would issue a "Rally" command signifying the end of the experimental trial and indicating that the Soldier was to teleoperate the TALON back to the mission start point.

\subsection{Data Collection}

\subsubsection{Data Processing}

The data for each participant were captured in the AMREL event data logs and by an experimental observer; however, the event data logs were incomplete, including complete sets of only chat and tactor data. Radio communications via the two-way radio was independent of the data logging mechanism. The event data logs recorded with millisecond precision represented a potentially more accurate source of target times than the records of the experimental observer, but the records kept by the experimental observer were complete for all three conditions, even though no doubt subject to rounding bias due to the hand recorded event records. In order to ensure accurate target time data, in the conditions where the redundant data existed, the time to each target within each communication condition was examined for both the data logs and the observer records. The examination revealed near equivalent records with slightly shorter observer record times than data log times. Since the observer records were complete, consistent across all conditions, and nearly equivalent to the data logs in redundant conditions, the observer records were selected for the time analysis. Otherwise, the three modes would be compared using two different recording mechanisms: the AMREL for the chat and tactor data and the manual recording for the audio data, leaving open the possibility of a confound between type of recording and communication mode. In the observer records, there were 16 total instances of either missing or ambiguous mission data. Each event was isolated and triangulated with data log elapsed times, observer record elapsed times, and observer trial notes. Each of the 16 missing or ambiguous target times was corrected using time estimates across the three sources.

To ensure the communication frequency data was accurate, the number of communications per target within each communication condition was examined in both the data logs and the observer records, again in the conditions where the redundant data existed. The examination of communication frequencies revealed that records between the two sources closely matched in the chat and tactor conditions, but varied considerably in the radio condition. The only means of communicating in the chat and tactor conditions was via the tablet, and therefore, transmitted with the tablet, and as a result, captured in the logs. Consequently, the data logs had to be $100 \%$ accurate for these two conditions and were preserved for analysis. This was not the case for the radio condition. Further examination of the observer trial notes and observer records revealed 
that repeated radio commands were recorded in the observer records, but only a single instance of the corresponding command was recorded in the data logs. It was inferred that in instances when the verbal radio command had to be repeated several times by the commander, the commander only entered the command in the tablet once; perhaps due to the multitasking demand of using the radios and tablet simultaneously. This provided evidence that the data logs were likely less accurate in radio command frequency than the observer records and that the observer records for radio command frequency were a valid, reliable, and unbiased substitution for analysis.

\section{Analyses and Results}

\subsection{Time to Identify Targets}

\subsubsection{Data Screening and Selection}

The time data for each participant was first plotted by communications type and target type, and then visually inspected. It was observed that the majority of the outlying or extreme values existed for trials in excess of $150 \mathrm{~s}$. A review of the observer run sheets indicated that trials approximating or exceeding $150 \mathrm{~s}$ often involved errors of some kind. Example errors were communication failures, failure of AMREL dismounted controller software, TALON malfunctions, or simply participant confusion. The data did not meet normality assumptions, showing evidence of skew, kurtosis, and significant non-normality according to the ShapiroWilk test of normality. A log transformation reduced skew to two instances and kurtosis to three instances, and only one condition failed the test of normality; however, filtering data points in excess of 150 s reduced skew to 0 instances and kurtosis to two instances, and resulted in normally distributed data in all conditions. Due to better meeting the normality assumptions with the filtered data, analysis was carried out with this latter data set.

\subsubsection{Analysis}

A $3 \times 3$ repeated measures analysis of variance (ANOVA) was performed on the time-toidentify-targets data based on communication type and target type.

\subsubsection{Results}

The interaction of communications type $\mathrm{x}$ target type was not significant, $F(4,48)=1.01, p \leq .41$, $\eta_{p}{ }^{2}=0.08$. Communications type was not significant, $F(2,24)=1.3, p \leq .29, \eta_{p}{ }^{2}=0.1$. Target type was significant, suggesting an effect of the size of targets on RT, $F(2,24)=9.72, p \leq .001$,

$\eta_{p}{ }^{2}=0.45$. The mean time to identify for the large target was $M=66.99$ s, for the small target was $M=80.34 \mathrm{~s}$, and the medium target was $M=87.17 \mathrm{~s}$. Post-hoc tests using the Bonferroni adjustment for multiple comparisons revealed that Soldiers were significantly faster identifying 
the large targets versus the small or medium targets, but that the results for the small target did not differ from the medium target. The significant post-hoc results are shown in table 2.

Table 2. Post-hoc tests on time to identify (seconds) by target type.

\begin{tabular}{|c|c|c|c|c|c|}
\hline \multicolumn{2}{|c|}{} & Mean Diff & SE & Sig & d' \\
\hline Large target & Medium target & 13.35 & 3.71 & 0.01 & 0.93 \\
\hline Large target & Small target & 20.18 & 5.62 & 0.01 & 1.07 \\
\hline
\end{tabular}

\subsection{Number of Navigational Communications}

\subsubsection{Data Screening and Selection}

The number of navigational communications for each participant in each condition were plotted by target type and visually inspected. Visually nothing appeared out of normal range, but the data within conditions did show evidence of minor skew, kurtosis, and small departures from normality using the Shapiro-Wilk test of normality. A natural log transformation was performed on the data to eliminate these problems. The transformation corrected any normality violations. Log linear is similar to a chi square in that it can handle interactions, which makes it a nonparametric equivalent to the $3 \times 3$ parametric ANOVA concurrently performed on the data. The two complimentary analyses provide a more comprehensive evaluation of the data so that we could make a better judgment about patterns in the data. Additionally, since the communication data were frequency counts, they were also compiled as total conditional frequencies for a tabular analysis. The total number of communications was counted across all participants within each condition. Due to two small targets in each mission, the large and medium targets each had two repetitions across conditions, but the small target segment had four repetitions. To account for the extra repetitions for the small target, the total frequency was calculated and then divided by two to make the small target frequency equivalent relative to the large and medium targets frequencies.

\subsubsection{Analyses}

First, a $3 \times 3$ repeated measures ANOVA was performed on the navigational communications data with communication type and target type. Next, a log linear analysis was performed on the cross tabulated navigational communication frequency data.

\subsubsection{Results}

The interaction of communications type $x$ target type was not significant, $F(4,48)=1.23, p \leq .31$, $\eta_{p}{ }^{2}=0.09$. Communications type was not significant, $F(2,24)=1.89, p \leq .17, \eta_{p}{ }^{2}=0.14$. Target type was significant, suggesting an effect of the size of targets on the number of communications, $F(2,24)=10.99, p<.001, \eta_{p}{ }^{2}=.48$. Using raw frequencies, the mean count for the large target was $M=5.88$, for the small target was $M=7.48$, and for the medium target was $M=7.73$. Post-hoc tests using the Bonferroni adjustment for multiple comparisons revealed that Soldiers did require 
significantly fewer communications to identify the large target versus both the small and medium targets, but the small target results did not differ from the medium target results. The significant post-hoc results are shown in table 3 using the log transformed data.

Table 3. Communication count pos- hoc tests on target type.

\begin{tabular}{|c|c|c|c|c|c|}
\hline \multicolumn{2}{|c|}{} & Mean Diff & SE & Sig & d' \\
\hline Large target & Medium target & 0.30 & 0.07 & 0.002 & 1.35 \\
\hline Large target & Small target & 0.27 & 0.06 & 0.003 & 0.93 \\
\hline
\end{tabular}

The log linear analysis supported the results of the previous ANOVA. Starting with the saturated model, the final model kicked out the interaction term leaving only the first order effects. Of the two first order effects, target type clearly had the stronger impact on the model with a significant log likelihood chi square value nearly $3 \times$ as large as the likelihood value for communications type. The final model with both first order effects was not significant (using a strict alpha criterion of .05) $\chi^{2}(4,1648)=9.08, \mathrm{p} \leq .059$. However, the models with either target type or communications type alone were significant. The log likelihood chi square value $\chi^{2}(2,1648)=8.96, \mathrm{p} \leq .011$ for communications type reveals that fewer communications were observed in the tactor condition relative to the chat and radio conditions than were expected. The observed and expected frequencies are shown in table 4. Likewise, $\chi^{2}(2,1648)=22.09$, $\mathrm{p}<0.001$ for target type indicates that fewer communications were observed for the large target condition relative to the small and medium conditions than were expected.

Table 4. Communication frequencies.

\begin{tabular}{|l|c|c|c|}
\hline & Observed N & Expected N & Residual \\
\hline Tactor & 498 & 549.3 & -51.3 \\
\hline Chat & 597 & 549.3 & 47.7 \\
\hline Radio & 553 & 549.3 & 3.7 \\
\hline Total & 1648 & - & - \\
\hline
\end{tabular}

\subsection{Exploratory Analysis}

\subsubsection{Data Screening and Selection}

The subjective threshold that was noted regarding the 150-s ceiling for errors raised some questions about the data in hindsight. Research on human error (Horrey and Wickens, 2007) suggests that often the most important details regarding error can be in the tails of the distribution, and that, studies focusing on the centrality of the distributions may mask important evidence. Consequently, the upper and lower halves of the time-to-identify-targets and communication-frequency data sets were contrasted for differences. The median of each data set was computed and the data recoded as either above or below the median. 


\subsubsection{Analyses}

Chi square analyses were run on the median split data for time and communication frequency.

\subsubsection{Results}

The results of the chi square on the median split time data did not show evidence of a proportional difference in time to identify targets across communication types. The pattern of instances shown in table 5 reflects a greater proportion of trials below the median than above it in the tactor condition relative to the chat and radio conditions. This pattern was not inferentially supported though given $\chi^{2}(2,117)=0.27, \mathrm{p} \leq 0.87$, Cramer's V=0.05.

Table 5. Time to identify targets: median split.

\begin{tabular}{|c|c|c|c|c|}
\hline & Tactor & Chat & Radio & Total \\
\hline Below median & 21 & 19 & 19 & 59 \\
\hline Above median & 18 & 20 & 20 & 58 \\
\hline Total & 39 & 39 & 39 & 117 \\
\hline
\end{tabular}

The results of the median split on communication frequency did show evidence of a proportional difference in frequency of communications across communication type. The pattern of instances shown in table 6 reflects a greater proportion of trials below the median than above it in the tactor condition relative to the chat and radio conditions. The $\chi^{2}$ test did not show statistical significance at the conventional $\alpha=0.05$ level but this pattern did show evidence of inferential support given $\chi^{2}(2,117)=5.41, p \leq 0.067$, Cramer's V $=0.22$.

Table 6. Communication frequency median split.

\begin{tabular}{|c|c|c|c|c|}
\hline & Tactor & Chat & Radio & Total \\
\hline Below median & 26 & 16 & 19 & 61 \\
\hline Above median & 13 & 23 & 20 & 56 \\
\hline Total & 39 & 39 & 39 & 117 \\
\hline
\end{tabular}

\subsection{NASA TLX and SART Analyses}

\subsubsection{Data Screening and Selection}

Both the NASA TLX and SART data sets were screened for illegal values. None were found. The rating data revealed non-normality using the Shapiro-Wilk test of normality and consequently treated ordinally in the analysis to resolve the lack of normality.

\subsubsection{Analyses}

Both data sets were analyzed using the Friedman nonparametric test for dependent samples. Only the primary variable of interest, communication type, was used in this analysis. 


\subsubsection{Results}

None of the six dimensions of workload showed any evidence of differences between communication types. Likewise, none of the three dimensions of SA indicated any differences between communication types.

\section{Conclusion}

There was considerable noise in the data caused by intermittent problems with the TALON, among other uncontrollable factors. The power analysis allowed us to conclude that additional participants would not have changed the results. In particular, we found that communication mode has no real effect on target identification time, i.e., no mode performed significantly better or worse than the others. This in itself is an important finding because it suggests that the tactical use of haptic signals for covert communications may be feasible in light of finding no evidence to support differential performance within the haptic form of communication. Thus, haptics could potentially be a desirable tactical option. While not implying performance is equivalent among communication modes, we obtained no evidence that would lead us to believe the converse, i.e., there was a performance decrement for tactile communications. This is gratifying because of the difficulty of the task, which consisted of finding targets in a previously unknown environment using an unfamiliar tactile lexicon while navigating a TALON robot with which they had limited familiarity.

Gilson et al. (2007) suggested that robotic operators will be able to use low bandwidth tactile signals to communicate covertly with their chain of command and eventually communicate with future robots with enhanced cognitive abilities. Perhaps of equal importance is that tactile communications permit Soldiers to use a virtually unused communications channel to increase SA without impinging on their visual or auditory resources (Barnes, in press). Also, there was evidence that the tactile cueing might actually be an efficient means of communication, as reflected in the results that indicted a reduced number of communications were necessary for target location in this mode; however, this finding requires additional research for verification.

Also, it is important to note that the 13 Soldiers were relatively efficient in using the TALON to find targets in desert terrain. It would be a mistake to assume this was an easy task. The targets were hidden in the desert foliage so that the targets were never in plain sight of the robot sensor system. Also, during this time of year, there was a great deal of vegetation on the desert floor including complex sand dunes overgrown with thorny creosote bushes because of the monsoon rains. The training was minimal; total time was $>1 \mathrm{~h}$. The training took place in a separate area to ensure that Soldiers were not familiar with target locations. Nevertheless, the Soldiers were able to navigate the TALON to most of the targets rapidly. There was a consistent target effect. Logically, it would seem the small target would be the most difficult target to find and 
consequently reflect a great search time and/or communication frequency. This was not the case. Rather, the results showed evidence that medium targets were the most challenging to identify. In summary, the results reinforced evidence from previous research that tactile communications are a tactically useful form of communication for Soldier-robot interactions.

Past research has shown that the tactile channel is beneficial when used redundantly with either the auditory or visual channel. In these paired instances, a tactile cue may increase both speed and accuracy of Soldier responses (e.g., Hempel and Altinsoy, 2005). The current research suggests that in environmental manipulations that make normal communications modalities ineffective, the tactile channel may serve as a tactically effective alternative. The findings in this experiment suggest that the inclusion of the tactile mode for communication poses no detriment in performance, and perhaps even an enhancement. These results make a good argument to examine transitioning this communications channel to a variety of operations. Subsequent experimentation should include examining chat, radio, and tactile communication modes in day versus night or safe versus covert environments, and possibly further exploring tactile communications as a redundant channel within the various environments. 


\section{References}

Barnes, M. J. Soldier-Robot Teaming in Future Military Systems: An Overview. In HumanRobot Interactions in Future Military Operations, Barnes, M. J.; Jentsch, F. D., eds.; Ashgate Publishing: Hampshire, UK, in press.

Carafano, J. J.; Gudgel, A. The Pentagon’s Robots: Arming the Future. The Heritage Foundation: Backgrounder 2007, 2096, 1-6.

Chen, J.Y.C.; Haas, E. C.; Barnes, M. J. Human Performance Issues and User Interface Design for Teleoperated Robots. IEEE Transactions on Systems, Man, and Cybernetics--Part C: Applications and Reviews 2007, 37 (6), 1231-1245.

Chen, J.Y.C.; Joyner, C. T. Concurrent Performance of Gunner’ and Robotic Operator's Tasks in a Multi-Tasking Environment. Military Psychology 2009, 21 (1), 98-113.

Chen, J.Y.C.; Terrence, P. I. Effects of Tactile Cueing on Concurrent Performance of Military and Robotics Tasks in a Simulated Multi-Tasking Environment. Ergonomics 2008, 51 (8), 1137-1152.

Gilson, R. D.; Redden, E. S.; Elliott, L. R. Remote Tactile Displays for Future Soldier; ARLSR-0152; U.S. Army Research Laboratory: Aberdeen Proving Ground, MD, 2006.

Haas, E. C. The Role of Emerging Multimodal Technologies in Enhancing Combat and Civilian System Safety. Journal of the American Society of Safety Engineer 2007.

Hart, S. G.; Staveland, L. E. Development of NASA-TLX (Task Load Index): Results of Empirical and Theoretical Research. In Human Mental Workload, Hancock, A.; Meshkati, H., eds., Elsevier: Amsterdam, 1987.

Hempel, T.; Altinsoy, E. Multimedia/Multimodal Displays. In Handbook of Human Factors in Web Design, Proctor, R.W.; Vu, K. P., eds., Lawrence Erlbaum: London, 2005, pp 134-145.

Horrey, W.; Wickens, C. In-Vehicle Glance Duration. Distributions, Tails, and Model of Crash Risk. Journal of the Transportation Research Board, Transportation Research Board of the National Academies, Washington, D.C. 2007, 2018, pp 22-28.

Jones, L. A.; Sarter, N. B. Tactile Displays: Guidance for Their Design and Application. Human Factors 2008, 50 (1), 90-111.

Pettit, R. A.; Redden, E. S.; Carstens, C. C. Comparison of Hand and Arm Signals to a Covert Tactile Communication System in a Dynamic Environment; ARL-TR-3838; U.S. Army Research Laboratory: Aberdeen Proving Ground, MD, 2006. 
Taylor, R. M. Situational Awareness Rating Technique (SART): The Development of a Tool For Aircraft Systems Design. In Situational Awareness in Aerospace Operations. Neuilly Sur Seine, France: NATO-AGARD, 1990. AGARD-CP-478. 


\section{List of Symbols, Abbreviations, and Acronyms}

$\begin{array}{ll}\text { ANOVA } & \text { Analysis of Variance } \\ \text { ARL } & \text { U.S. Army Research Laboratory } \\ \text { CC-SART } & \text { Compatibility Situation Awareness Technique Questionnaire } \\ \text { ATO } & \text { U.S. Army Technology Objective } \\ \text { FCS } & \text { Future Combat System } \\ \text { HRI } & \text { human robot interaction } \\ \text { HRED } & \text { Human Research and Engineering Directorate } \\ \text { NASA-TLX } & \text { National Aeronautics and Space Administration-Task Load Index } \\ \text { RC } & \text { Robotics Collaboration } \\ \text { SA } & \text { situation awareness } \\ \text { SUGV } & \text { small unmanned ground vehicle } \\ \text { TARDEC } & \text { U.S. Army Tank-Automotive Research Development and Engineering Center } \\ \text { UAVs } & \text { unmanned air vehicles } \\ \text { UGVs } & \text { unmanned ground vehicles }\end{array}$


NO. OF

COPIES ORGANIZATION

\begin{tabular}{|c|c|}
\hline 1 & DEFENSE TECHNICAL \\
\hline (PDF & INFORMATION CTR \\
\hline & DTIC OCA \\
\hline & 8725 JOHN J KINGMAN RD \\
\hline & STE 0944 \\
\hline & FORT BELVOIR VA 22060-6218 \\
\hline 1 & DIRECTOR \\
\hline & US ARMY RESEARCH LAB \\
\hline & IMNE ALC HRR \\
\hline & 2800 POWDER MILL RD \\
\hline & ADELPHI MD 20783-1197 \\
\hline 1 & DIRECTOR \\
\hline & US ARMY RESEARCH LAB \\
\hline & RDRL CIM L \\
\hline & 2800 POWDER MILL RD \\
\hline & ADELPHI MD 20783-1197 \\
\hline 1 & DIRECTOR \\
\hline & US ARMY RESEARCH LAB \\
\hline & RDRL CIM P \\
\hline & 2800 POWDER MILL RD \\
\hline & ADELPHI MD 20783-1197 \\
\hline 1 & DIRECTOR \\
\hline & US ARMY RESEARCH LAB \\
\hline & RDRL D \\
\hline & 2800 POWDER MILL RD \\
\hline & ADELPHI MD 20783-1197 \\
\hline & ABERDEEN PROVING GROUND \\
\hline 1 & DIR USARL \\
\hline & RDRL CIM \\
\hline
\end{tabular}


NO. OF

COPIES ORGANIZATION

1 ARMY RSCH LABORATORY - HRED

RDRL HRM A J MARTIN

MYER CENTER BLDG 2700 RM 2D311

FORT MONMOUTH NJ 07703-5601

1 ARMY RSCH LABORATORY - HRED RDRL HRM C A DAVISON 320 MANSCEN LOOP STE 115 FORT LEONARD WOOD MO 65473

1 ARMY RSCH LABORATORY - HRED RDRL HRM DI T DAVIS

BLDG 5400 RM C242

REDSTONE ARSENAL AL 35898-7290

1 ARMY RSCH LABORATORY - HRED RDRL HRS EA DR V J RICE

BLDG 4011 RM 217

1750 GREELEY RD

FORT SAM HOUSTON TX 78234-5002

1 ARMY RSCH LABORATORY - HRED RDRL HRM DG K GUNN

BLDG 333

PICATINNY ARSENAL NJ 07806-5000

1 ARMY RSCH LABORATORY - HRED ARMC FIELD ELEMENT RDRL HRM CH C BURNS THIRD AVE BLDG 1467B RM 336 FORT KNOX KY 40121

1 ARMY RSCH LABORATORY - HRED AWC FIELD ELEMENT RDRL HRM DJ D DURBIN BLDG 4506 (DCD) RM 107 FORT RUCKER AL 36362-5000

1 ARMY RSCH LABORATORY - HRED RDRL HRM CK J REINHART 10125 KINGMAN RD

FORT BELVOIR VA 22060-5828

1 ARMY RSCH LABORATORY - HRED RDRL HRM AY M BARNES 2520 HEALY AVE STE 1172 BLDG 51005 FORT HUACHUCA AZ 85613-7069

1 ARMY RSCH LABORATORY - HRED RDRL HR MP D UNGVARSKY POPE HALL BLDG 470 BCBL 806 HARRISON DR FORT LEAVENWORTH KS 66027-2302
NO. OF

COPIES ORGANIZATION

1 ARMY RSCH LABORATORY - HRED RDRL HRM AJ J HANSBERGER JFCOM FE 115 LAKEVIEW PKWY STE B SUFFOLK VA 23435

1 ARMY RSCH LABORATORY - HRED RDRL HRM DQ M R FLETCHER NATICK SOLDIER CTR AMSRD NSC WS E BLDG 3 RM 343 NATICK MA 01760-5020

1 ARMY RSCH LABORATORY - HRED RDRL HRM AT J CHEN 12350 RESEARCH PKWY ORLANDO FL 32826-3276

1 ARMY RSCH LABORATORY - HRED RDRL HRM AT C KORTENHAUS 12350 RESEARCH PKWY ORLANDO FL 32826

1 ARMY RSCH LABORATORY - HRED RDRL HRM AS C MANASCO SIGNAL TOWERS BLDG 29808A RM 303A FORT GORDON GA 30905-5233

1 ARMY RSCH LABORATORY - HRED RDRL HRM CU 6501 E 11 MILE RD MS 284 BLDG 200A 2ND FL RM 2104 WARREN MI 48397-5000

1 ARMY RSCH LABORATORY - HRED FIRES CTR OF EXCELLENCE FIELD ELEMENT RDRL HRM AF C HERNANDEZ 3040 NW AUSTIN RD RM 221 FORT SILL OK 73503-9043

1 ARMY RSCH LABORATORY - HRED RDRL HRM AV S MIDDLEBROOKS 91012 STATION AVE RM 348 FORT HOOD TX 76544-5073

1 ARMY RSCH LABORATORY - HRED RDRL HRM CN R SPENCER DCSFDI HF HQ USASOC BLDG E2929 FORT BRAGG NC 28310-5000 
NO. OF

COPIES ORGANIZATION

1 ARMY RSCH LABORATORY - HRED

RDRL HRM DW E REDDEN

BLDG 4 CL 60

FORT BENNING GA 31905-5400

1 ARMY G1

(CD DAPE MR B KNAPP

only) 300 ARMY PENTAGON RM 2C489

WASHINGTON DC 20310-0300

\section{$\underline{\text { ABERDEEN PROVING GROUND }}$}

5 DIR USARL

RDRL CIM G

S FOPPIANO

RDRL HR

L ALLENDER

T LETOWSKI

RDRL HRM B

J LOCKETT

RDRL HRS D

B AMREIN 
INTENTIONALLY LEFT BLANK. 